\title{
An Explicit Analytic Solution to the Thomas-Fermi Equation by the Improved Differential Transform Method
}

\author{
H. FATOOREHCHI ${ }^{a}$ AND H. ABOLGHASEMI ${ }^{a, b, *}$ \\ ${ }^{a}$ Center for Separation Processes Modeling and Nano-Computations, School of Chemical Engineering \\ College of Engineering, University of Tehran, P.O. Box 11155/4563, Tehran, Iran \\ ${ }^{b}$ Oil and Gas Center of Excellence, University of Tehran, Tehran, Iran \\ (Received March 29, 2013; revised version September 27, 2013; in final form February 20, 2014) \\ In this paper, a newly proposed analytical scheme by the authors namely the improved differential transform \\ method is employed to provide an explicit series solution to the Thomas-Fermi equation. The solution procedure \\ is very straightforward, requiring merely elementary operations together with differentiation, and ends up in a \\ recursive formula involving the Adomian polynomials to afford the unknown coefficients. Unlike many other \\ methods, our approach is free of integration and hence can be of computational interest. In addition, a very \\ good agreement between the proposed solution and the results from several well-known works in the literature is \\ demonstrated.
}

DOI: 10.12693/APhysPolA.125.1083

PACS: 02.30.Hq, 02.30.Mv, 02.60.Lj, 21.10.Ft, 31.15.-p

\section{Introduction}

In quantum mechanics, the Thomas-Fermi equation is a well-known nonlinear ordinary differential equation (ODE) which affords the electrostatic potential associated with the Thomas-Fermi atom model. The equation, in its normalized form, reads

$$
\frac{\mathrm{d}^{2} u}{\mathrm{~d} x^{2}}=\frac{\sqrt{u^{3}}}{\sqrt{x}}
$$

subject to the following boundary conditions:

$$
u(0)=1 \text { and } u(+\infty)=0 .
$$

It is easily noticed that the Thomas-Fermi equation has a singular point at $x=0$. Kobayashi et al. [1] were of the early researchers to numerically analyze the ThomasFermi equation. As another numerical treatment of the Thomas-Fermi equation, MacLeod [2] used the Chebyshev series technique for small and large quantities of the independent variable $x$. A variety of analytical approximate solutions have been provided by different approaches namely the $\delta$-expansion method [3], the Adomian decomposition method $[4,5]$, the homotopy analysis method [6-9], and the modified variational iteration method [10].

The aim of this paper is to present a new explicit analytical solution for the Thomas-Fermi equation which is based on a recently developed scheme by the authors viz. the improved differential transform method (IDTM) [11] combined with the Padé approximants to treat the boundary condition at the infinity.

*corresponding author; e-mail: abolghasemi.ha@gmail.com, hoab@ut.ac.ir

\section{Preliminaries of the improved differential transform method}

The differential transform method (DTM) was originally conceptualized by Zhou during his studies on electrical circuits [12]. The method yields analytical solutions in form of polynomial series through a straightforward manner: by taking the differential of a functional equation which may be differential, integral or integro-differential, and performing elementary operations, one can construct a recurrence within the transformed domain. Afterwards, the exact analytical solution would readily be achieved by utilization of the simply defined inverse differential transform. The other salient merits of the DTM include its not requiring discretization, linearization or perturbation [13]. Many researchers have exploited the DTM to solve both linear and nonlinear functional equations, yet limited to power-law and integral product nonlinearity types, in applied mathematics, physics, aerodynamics, chemical engineering and heat transfer, just to mention a few areas [14-22].

The one-dimensional differential transform of a given univariate function $u(x)$ is defined by

$$
U(k)=D T\{u(x)\}=\frac{1}{k !}\left[\frac{\mathrm{d}^{k} u(x)}{\mathrm{d} x^{k}}\right]_{x=0},
$$

where $u(x)$ and $U(k)$ denote the original and transformed functions, orderly.

Correspondingly, the inverse differential transform is introduced by

$$
u(x)=D T^{-1}\{U(k)\}=\sum_{k=0}^{\infty}\left\{U(k) x^{k}\right\} .
$$

Obviously, $D T\{\cdot\}$ and $D T^{-1}\{\cdot\}$ designate the direct and inverse differential transform operators in the definitions above, respectively. Regarding the preceding equation, one can immediately recognize the simplicity inher- 
ent in the definition of the inverse differential transform. We skip the details on the definitions/properties of the $n$-dimensional differential transform and refer the interested reader to [14]. A number of fundamental operations of the one-dimensional differential transform are given in Appendix A, Table A.1.

Most recently, Fatoorehchi and Abolghasemi [11] have managed to extend the DTM to nonlinear functional equations of arbitrary types. The essence of this extended method, dubbed the IDTM, is reflected in the following theorem.

Theorem 2.1. Let $U(k)=D T\{u(x)\}$ and consequently $u(x)=D T^{-1}\{U(k)\}$. It holds true that,

$$
D T\{N(u)\}=A_{k}(U(0), U(1), \ldots, U(k)),
$$

where $A_{k}$ represent the Adomian polynomials related to the nonlinear operator $N$ and are classically defined by

$$
A_{k}\left(U_{0}, U_{1}, \ldots, U_{k}\right)=\frac{1}{k !}\left[\frac{\mathrm{d}^{k}}{\mathrm{~d} \lambda^{k}} N\left(\sum_{i=0}^{\infty} \lambda^{i} U_{i}\right)\right]_{\lambda=0} .
$$

Proof: The proof to this theorem is fully covered in [11].

The respected reader who is new to the Adomian polynomials can consult Refs. [23-28] for background information.

Additionally, the theorem to follow often comes in handy in analysis of functional equations.

Theorem 2.2. Given $D T\{f(t)\}=F(k)$, it holds for any non-negative integer $m$ that

$$
D T\left\{t^{m} f(t)\right\}=\left\{\begin{array}{l}
F(k-m), \quad k \geq m, \\
0, \quad 0 \leq k<m .
\end{array}\right.
$$

Proof: Substitute $u(t)=f(t)$ and $v(t)=t^{m}$ into the fourth property and then utilize the fifth property stated in Table I to yield

$$
D T\left\{t^{m} f(t)\right\}=\sum_{i=0}^{k}\{F(i) \delta(k-i-m)\} .
$$

According to the definition of the Kronecker delta function, all components of the preceding summation vanish except for the one with $i=k-m$. Since $i \geq 0$, the following equation is valid for $k-m \geq 0$ or $k \geq m$ :

$$
D T\left\{t^{m} f(t)\right\}=F(k-m) .
$$

To obtain the differential transform of $t^{m} f(t)$ for $0 \leq k<m$, we shall refer to Eq. (3),

$$
D T\left\{t^{m} f(t)\right\}=\frac{1}{k !}\left[\frac{\mathrm{d}^{k}\left(t^{m} f(t)\right)}{\mathrm{d} t^{k}}\right]_{t=0} .
$$

By the general Leibniz rule it is easily yielded that

$$
D T\left\{t^{m} f(t)\right\}=0 ; \quad 0 \leq k<m .
$$

Equation (9) together with (11) concludes the proof.

\section{Solution of the Thomas-Fermi equation by the IDTM}

\begin{tabular}{|c|c|}
\hline Original function & Transformed function \\
\hline$u(x) \pm v(x)$ & $U(k) \pm V(k)$ \\
\hline$\alpha u(x)$ & $\alpha U(k)$ \\
\hline$\frac{\mathrm{d}^{m} u(x)}{\mathrm{d} x^{m}}$ & $\frac{(k+m) !}{k !} U(k+m)$ \\
\hline$u(x) v(x)$ & $\sum_{r=0}^{k}\{U(r) V(k-r)\}$ \\
\hline$x^{m}$ & $=\left\{\begin{array}{l}1, \quad k=m \\
0, k \neq m\end{array}\right.$ \\
\hline $\mathrm{e}^{a x}$ & $\frac{a^{k}}{k !}$ \\
\hline $\sin (a x)$ & $\frac{a^{k}}{k !} \sin \left(\frac{k \pi}{2}\right)=\left\{\begin{array}{l}0, k \in \text { even }, \\
\frac{a^{k}(-1) \frac{k-1}{2}}{k !}, k \in \text { odd }\end{array}\right.$ \\
\hline $\cos (a x)$ & $\frac{a^{k}}{k !} \cos \left(\frac{k \pi}{2}\right)=\left\{\begin{array}{l}\frac{a^{k}(-1)^{\frac{k}{2}}}{k !}, k \in \text { even } \\
0, k \in \text { odd }\end{array}\right.$ \\
\hline$f(x)=\int_{x_{0}}^{x} u(t) \mathrm{d} t$ & $F(k)=\frac{U(k-1)}{k}, k \geq 1 ; F(0)=0$ \\
\hline$f(x)=\int_{x_{0}}^{x} g(t) u(t) \mathrm{d} t$ & $F(k)=\sum_{l=0}^{k-1}\left\{G[l] \frac{U(k-l-1)}{k}\right\}, k \geq 1 ; F(0)=0$ \\
\hline
\end{tabular}

For the sake of simplicity and to get rid of the fractional order of the Thomas-Fermi equation, firstly we let $t=\sqrt{x}$.
TABLE I

Some basic operations of the one-dimensional differential transform.

So,

$$
\begin{aligned}
\frac{\mathrm{d}^{2} u}{\mathrm{~d} x^{2}} & =\frac{\mathrm{d}}{\mathrm{d} t}\left(\frac{\mathrm{d} u}{\mathrm{~d} x}\right) \frac{\mathrm{d} t}{\mathrm{~d} x}=\frac{\mathrm{d}}{\mathrm{d} t}\left(\frac{1}{2 t} \frac{\mathrm{d} u}{\mathrm{~d} t}\right) \frac{1}{2 t} \\
= & \left(\frac{1}{2 t} \frac{\mathrm{d}^{2} u}{\mathrm{~d} t^{2}}-\frac{1}{2 t^{2}} \frac{\mathrm{d} u}{\mathrm{~d} t}\right) \frac{1}{2 t}=\frac{1}{4 t^{2}} \frac{\mathrm{d}^{2} u}{\mathrm{~d} t^{2}}-\frac{1}{4 t^{3}} \frac{\mathrm{d} u}{\mathrm{~d} t} .
\end{aligned}
$$

Therefore, Eq. (1) is converted to

$$
\frac{1}{4 t^{2}} \frac{\mathrm{d}^{2} u}{\mathrm{~d} t^{2}}-\frac{1}{4 t^{3}} \frac{\mathrm{d} u}{\mathrm{~d} t}=\frac{u^{\frac{3}{2}}}{t},
$$

or equally,

$$
t \frac{\mathrm{d}^{2} u}{\mathrm{~d} t^{2}}-\frac{\mathrm{d} u}{\mathrm{~d} t}=4 t^{2} u^{\frac{3}{2}}
$$

Taking the differential transform of Eq. (14) gives

$$
D T\left\{t \frac{\mathrm{d}^{2} u}{\mathrm{~d} t^{2}}\right\}-(k+1) U(k+1)=4 D T\left\{t^{2} u^{\frac{3}{2}}\right\} .
$$

Letting $D T\left\{u^{\frac{3}{2}}\right\}=F(k)$, we obtain the following from Theorem 2.1:

$$
\begin{aligned}
& F(0)=[U(0)]^{\frac{3}{2}}, \\
& F(1)=\frac{3}{2}[U(0)]^{\frac{1}{2}} U(1), \\
& F(2)=\frac{3}{8} \frac{[U(1)]^{2}}{[U(0)]^{\frac{1}{2}}}+\frac{3}{2}[U(0)]^{\frac{1}{2}} U(2), \\
& F(3)=-\frac{1}{16} \frac{[U(1)]^{3}}{[U(0)]^{\frac{3}{2}}}+\frac{3}{4} \frac{U(1) U(2)}{[U(0)]^{\frac{1}{2}}}+\frac{3}{2}[U(0)]^{\frac{1}{2}} U(3) \\
& \quad:
\end{aligned}
$$

By invoking Theorem 2.2, Eq. (15) leads to

$$
k(k+1) U(k+1)-(k+1) U(k+1)=4 F(k-2)
$$

or

$$
U(k+1)=\frac{4}{k^{2}-1} F(k-2)
$$

for $k \geq 2$.

Also, for the case with $k=0$, we get $U(1)=0$, and obviously, $k=1$ leads to the trivial result $0=0$. This 
is not far from expectation as our solution shall contain one free parameter to be fixed by the second boundary condition, which is the one prescribed at the infinity.

Moreover, the boundary condition at zero easily determines that $U(0)=1$.

Thus, it follows from Eq. (18) that

$$
\begin{aligned}
& U(3)=\frac{4}{3} F(0), U(4)=\frac{1}{2} F(1), U(5)=\frac{4}{15} F(2), \\
& U(6)=\frac{1}{6} F(3), U(7)=\frac{4}{35} F(4), U(8)=\frac{1}{12} F(5), \\
& U(9)=\frac{4}{63} F(6), \ldots
\end{aligned}
$$

As discussed above $U(2)$ cannot be determined by the foregoing equations, so we set $U(2)=\alpha$ and later will determine it by the help of the boundary condition at the infinity.

In view of prior equations, we find

$$
\begin{aligned}
& U(0)=1, U(1)=0, U(2)=\alpha, U(3)=\frac{4}{3}, U(4)=0, \\
& U(5)=\frac{2}{5} \alpha, U(6)=\frac{1}{3}, U(7)=\frac{3}{70} \alpha^{2}, U(8)=\frac{2}{15} \alpha, \\
& U(9)=-\frac{\alpha^{3}}{252}+\frac{2}{27}, \ldots
\end{aligned}
$$

Finally, we can propose the solution to Eq. (14) as

$$
\begin{aligned}
& u(t)=\sum_{k=0}^{\infty}\left\{U(k) t^{k}\right\}=1+\alpha t^{2}+\frac{4}{3} t^{3}+\frac{2}{5} \alpha t^{5}+\frac{1}{3} t^{6} \\
& +\frac{3}{70} \alpha^{2} t^{7}+\frac{2}{15} \alpha t^{8}+\left(-\frac{\alpha^{3}}{252}+\frac{2}{27}\right) t^{9}+\frac{1}{175} \alpha^{2} t^{10} \\
& +\left(\frac{1}{1056} \alpha^{4}+\frac{31}{1485} \alpha\right) t^{11}+\left(\frac{4}{1575} \alpha^{3}+\frac{4}{405}\right) t^{12} \\
& -\left(\frac{3}{9152} \alpha^{5}-\frac{557}{100100} \alpha^{2}\right) t^{13}-\left(\frac{29}{24255} \alpha^{4}\right. \\
& \left.-\frac{4}{693} \alpha\right) t^{14}+\left(\frac{7}{49920} \alpha^{6}-\frac{623}{351000} \alpha^{3}\right. \\
& \left.+\frac{101}{52650}\right) t^{15}+\left(\frac{68}{105105} \alpha^{5}-\frac{46}{45045} \alpha^{2}\right) t^{16} \\
& +\left(\frac{3}{43520} \alpha^{7}-\frac{153173}{116424000} \alpha^{4}+\frac{113}{1178100} \alpha\right) t^{17} \\
& -\left(\frac{4}{10395} \alpha^{6}-\frac{1046}{675675} \alpha^{3}-\frac{23}{473850}\right) t^{18} \\
& -\left(\frac{1232941}{1278076800} \alpha^{5}-\frac{799399}{698377680} \alpha^{2}\right) t^{19} \\
& +\left(\frac{256}{1044225} \alpha^{7}-\frac{99856}{70945875} \alpha^{4}+\frac{51356}{103378275} \alpha\right) t^{20} \\
& -\left(\frac{143}{6537216} \alpha^{9}-\frac{705965027}{966226060800} \alpha^{6}\right. \\
& \left.+\frac{33232663}{25881055200} \alpha^{3}-\frac{35953}{378132300}\right) t^{21}
\end{aligned}
$$

$$
\begin{aligned}
& -\left(\frac{6272}{38105925} \alpha^{8}-\frac{43468}{33622875} \alpha^{5}+\frac{250054}{342953325} \alpha^{2}\right) t^{22} \\
& +\left(\frac{143}{10551296} \alpha^{10}-\frac{4524629159}{7953566100480} \alpha^{7}\right. \\
& \left.+\frac{1861464749}{1253187936000} \alpha^{4}-\frac{22773977}{95108013000} \alpha\right) t^{23} \\
& +\left(\frac{2048}{17782765} \alpha^{9}-\frac{14756758}{12455257815} \alpha^{6}\right. \\
& \left.+\frac{27134428}{23880381525} \alpha^{3}-\frac{823}{23108085}\right) t^{24} \\
& -\left(\frac{663}{75366400} \alpha^{11}-\frac{144926432597}{319825938432000} \alpha^{8}\right. \\
& \left.+\frac{2383837819589}{1455090436800000} \alpha^{5}-\frac{17319117797}{30580884180000} \alpha^{2}\right) t^{25} \\
& +\mathrm{O}\left(t^{26}\right) .
\end{aligned}
$$

We shall remark that the series solutions reported by Wazwaz [5] and Noor et al. [10] miss one term in the coefficient of $t^{20}$, namely (256/1044225) $\alpha^{7}$ unlike Eq. (21). This fact does not imply that the previous results are not correct, however, they have been not reported completely. In other words, if we continue the schemes described in [5] and [10] for further solution components, then the term $(256 / 1044225) \alpha^{7} t^{20}$ will appear.

\section{The Padé approximants}

A Padé approximant, symbolized by $[m / n]$, to the function $f(x)$ is a rational function defined by

$$
\begin{aligned}
& {[m / n]=R(x)} \\
& \quad=\frac{P(x)}{Q(x)}=\frac{p_{0}+p_{1} x+p_{2} x^{2}+\ldots+p_{m} x^{m}}{1+q_{1} x+q_{2} x^{2}+\ldots+q_{n} x^{n}},
\end{aligned}
$$

where the numerator and the denominator have no common factors, $m, n \geq 0$ and

$$
\begin{aligned}
& f(0)=R(0), \\
& f^{\prime}(0)=R^{\prime}(0), \\
& f^{\prime \prime}(0)=R^{\prime \prime}(0) \\
& \cdots \\
& f^{(m+n)}(0)=R^{(m+n)}(0) .
\end{aligned}
$$

In other words, if we expand the function $f(x)$ and $R(x)$ in their Maclaurin series, their first $(m+n+1)$ terms cancel each other out.

By matching the powers in the equation $Q(x) f_{\mathrm{T}}(x)=$ $P(x)+\mathrm{O}\left(x^{m+n+1}\right)$, where $f_{\mathrm{T}}(x)$ denotes the Maclaurin expansion of $f(x)$, one can derive the coefficients $p_{0}, \ldots, p_{m}$ and $q_{1}, \ldots, q_{n}$ by solving a system of linear algebraic equations $[29,30]$. In general, it is stated that recurrence relations such as Wynn's epsilon algorithm is more computationally efficient than solving the mentioned matrix problem in determination of the Padé approximants [31]. A classic and most comprehensive 
monograph on the basics of the Padé approximants is due to Barker [29].

As the convergence radius of a non-infinite polynomial series is not sufficiently large to contain the boundary conditions at an infinite or semi-infinite domain, we have to employ the Padé approximants to covert Eq. (21) to its equivalent rational function of polynomials in order for calculating the free parameter $\alpha$. As suggested by Wazwaz [5] the diagonal $[n / n]$ Padé approximants are the most suitable for this purpose. It is worthwhile to mention that the Padé approximants of a desired polynomial series can easily be evaluated by the help of the built-in routines available in the mathematical software packages like Maple or Mathematica.

The initial slope of the Thomas-Fermi potential, $\alpha=\mathrm{d} u(0) / \mathrm{d} x$,

TABLE II obtained from different Padé approximants.

\begin{tabular}{c|c|c|c|c|c}
\hline \hline $\begin{array}{c}\text { Padé } \\
\text { approximants }\end{array}$ & Present work & Wazwaz [5] & Yao [6] & Liao [8] & Khan and Xu [9] \\
\hline$[2 / 2]$ & -1.211413728 & -1.211413729 & $\mathrm{~N} / \mathrm{A}$ & $\mathrm{N} / \mathrm{A}$ & $\mathrm{N} / \mathrm{A}$ \\
{$[4 / 4]$} & -1.550525918 & -1.550525919 & $\mathrm{~N} / \mathrm{A}$ & $\mathrm{N} / \mathrm{A}$ & $\mathrm{N} / \mathrm{A}$ \\
{$[5 / 5]$} & -1.586834762 & $\mathrm{~N} / \mathrm{A}$ & -1.573319118 & -1.50419 & -1.542791808 \\
{$[7 / 7]$} & -1.586021034 & -1.586021037 & $\mathrm{~N} / \mathrm{A}$ & $\mathrm{N} / \mathrm{A}$ & $\mathrm{N} / \mathrm{A}$ \\
{$[8 / 8]$} & -1.588076818 & -1.588076820 & $\mathrm{~N} / \mathrm{A}$ & $\mathrm{N} / \mathrm{A}$ & $\mathrm{N} / \mathrm{A}$ \\
{$[10 / 10]$} & -1.588069580 & -1.588076779 & -1.576999604 & -1.54600 & -1.573824678 \\
{$[12 / 12]$} & -1.589115463 & $\mathrm{~N} / \mathrm{A}$ & $\mathrm{N} / \mathrm{A}$ & $\mathrm{N} / \mathrm{A}$ & $\mathrm{N} / \mathrm{A}$ \\
{$[14 / 14]$} & -1.586499424 & $\mathrm{~N} / \mathrm{A}$ & $\mathrm{N} / \mathrm{A}$ & $\mathrm{N} / \mathrm{A}$ & $\mathrm{N} / \mathrm{A}$ \\
{$[15 / 15]$} & -1.570718095 & $\mathrm{~N} / \mathrm{A}$ & -1.582810904 & -1.56437 & -1.579528916
\end{tabular}

Using the diagonal Padé approximants and the second boundary condition we have computed and compared the values of the free parameter $\alpha$ as summarized in Table II.

Figure 1 depicts the variation of Padé approximants of the potential function $u(t)$ with respect to the independent spatial variable $t$. It can be observed that a more accurate solution, which shall satisfy $u(+\infty)=0$, is obtained once the degrees of the Padé approximants are increased. The Padé approximant $[4,4]$ is found to yield reliable solutions only in the vicinity of the origin, i.e. $t<1$, as it converges to 1.717618793 for large values of $t$.

\section{Conclusion}

The IDTM was employed to develop an analytical solution to the Thomas-Fermi atom model. To put it briefly, the IDTM exploits the Adomian polynomials to enable the differential transform of nonlinear terms. As the final step, diagonal Padé approximants were used to make the solution satisfy the boundary condition at infinity, $u(+\infty)=0$. It was shown that, unlike the previous works, our approach is free of integration and requires simple arithmetic operations together with differentiation. Compared to a number of well-known results in the literature $[5,6,8,9]$, the proposed method was revealed to be highly accurate and efficient. It can be concluded that the IDTM, thanks to its mentioned merits, holds the promise to become of interest in analytical treatment of nonlinear equations of various types in the future.

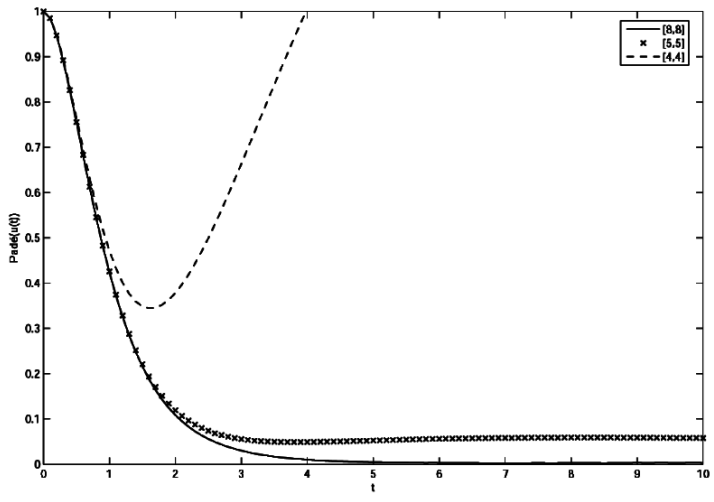

Fig. 1. Padé approximants $[4,4],[5,5]$ and $[8,8]$ of the potential function $u(t)$.

\section{Acknowledgments}

We, H.F. and H.A., would like to express our sincere gratitude to the reviewers and editor of the Acta Physica Polonica $A$ whose comments have indeed improved the quality of this contribution.

\section{References}

[1] S. Kobayashi, T. Matsukuma, S. Nagai, K. Umeda, J. Phys. Soc. Jpn. 10, 759 (1955).

[2] A.J. MacLeod, Comput. Phys. Commun. 67, 389 (1992).

[3] C.M. Bender, K.A. Milton, S.S. Pinsky, L.M. Simmons Jr., J. Math. Phys. 30, 1447 (1989). 
[4] G. Adomian, Appl. Math. Lett. 11, 131 (1998).

[5] A.-M. Wazwaz, Appl. Math. Comput. 105, 11 (1999).

[6] B. Yao, Appl. Math. Comput. 203, 396 (2008).

[7] A. El-Nahhas, Acta Phys. Pol. A 114, 913 (2008).

[8] S.J. Liao, Beyond Perturbation-Introduction to the Homotopy Analysis Method, Chapman and Hall, Boca Raton 2003.

[9] H. Khan, H. Xu, Phys. Lett. 365, 111 (2007).

[10] M.A. Noor, S.T. Mohyud-Din, M. Tahir, World Appl. Sci. J. 4, 479 (2008).

[11] H. Fatoorehchi, H. Abolghasemi, Appl. Math. Model. 37, 6008 (2013).

[12] J.K. Zhou, Differential Transformation and its Applications for Electrical Circuits, Huazhong University Press, Wuhan, China 1986 (in Chinese)

[13] F. Ayaz, Appl. Math. Comput. 143, 361 (2003).

[14] A. Kurnaz, G. Oturnaç, M.E. Kiris, Int. J. Comput. Math. 82, 369 (2005).

[15] Z.M. Odibat, Math. Comput. Model. 48, 1144 (2008).

[16] I.H. Abdel-Halim Hassan, Appl. Math. Model. 32, 2552 (2008).

[17] H. Fatoorehchi, H. Abolghasemi, Math. Comput. Model. 56, 145 (2012).

[18] A.S.V. Ravi Kanth, K. Aruna, Chaos Solitons Fractals 41, 2277 (2009).
[19] S. Hesam, A.R. Nazemi, A. Haghbin, Scientia Iranica 19, 1140 (2012).

[20] Y. Lien-Tsai, C. Cha'o-Kuang, Math. Comput. Model. 28, 101 (1998).

[21] H. Fatoorehchi, H. Abolghasemi, Aust. J. Basic Appl. Sci. 5, 337 (2011).

[22] C.W. Bert, J. Heat Transf. 124, 208 (2002).

[23] R. Rach, J. Math. Anal. Appl. 102, 415 (1984).

[24] G. Adomian, Solving Frontier Problems of Physics: The Decomposition Method, Kluwer Academic, Dordrecht 1994.

[25] J. Biazar, E. Babolian, G. Kember, A. Nouri, R. Islam, Appl. Math. Comput. 138, 523 (2003).

[26] E. Babolian, Sh. Javadi, Appl. Math. Comput. 153, 253 (2004).

[27] R. Rach, Kybernetes 37, 910 (2008).

[28] J.-S. Duan, Appl. Math. Comput. 216, 1235 (2010).

[29] G.A. Baker, Essentials of Padé Approximants, Academic Press, London 1975.

[30] M.A. Noor, S.T. Mohyud-Din, Comput. Math. Appl. 58, 2182 (2009).

[31] J.P. Boyd, Comput. Phys. 11, 299 (1997). 